Bull. Korean Math. Soc. 51 (2014), No. 2, pp. 423-435

http://dx.doi.org/10.4134/BKMS.2014.51.2.423

\title{
BOUNDEDNESS FOR FRACTIONAL HARDY-TYPE OPERATOR ON HERZ-MORREY SPACES WITH VARIABLE EXPONENT
}

\author{
JIANGLONG WU
}

\begin{abstract}
In this paper, the fractional Hardy-type operator of variable order $\beta(x)$ is shown to be bounded from the Herz-Morrey spaces $M \dot{K}_{p_{1}, q_{1}(\cdot)}^{\alpha, \lambda}\left(\mathbb{R}^{n}\right)$ with variable exponent $q_{1}(x)$ into the weighted space $M \dot{K}_{p_{2}, q_{2}(\cdot)}^{\alpha, \lambda}\left(\mathbb{R}^{n}, \omega\right)$, where $\omega=(1+|x|)^{-\gamma(x)}$ with some $\gamma(x)>0$ and $1 / q_{1}(x)-1 / q_{2}(x)=\beta(x) / n$ when $q_{1}(x)$ is not necessarily constant at infinity. It is assumed that the exponent $q_{1}(x)$ satisfies the logarithmic continuity condition both locally and at infinity that $1<q_{1}(\infty) \leq$ $q_{1}(x) \leq\left(q_{1}\right)_{+}<\infty\left(x \in \mathbb{R}^{n}\right)$.
\end{abstract}

\section{Introduction}

Let $f$ be a locally integrable function on $\mathbb{R}^{n}$. The $n$-dimensional Hardy operator is defined by

$$
\mathscr{H}(f)(x):=\frac{1}{|x|^{n}} \int_{|t|<|x|} f(t) \mathrm{d} t, \quad x \in \mathbb{R}^{n} \backslash\{0\} .
$$

In 1995, Christ and Grafakos [2] obtained the result for the boundedness of $\mathscr{H}$ on $L^{p}\left(\mathbb{R}^{n}\right)(1<p<\infty)$ spaces, and they also found the exact operator norms of $\mathscr{H}$ on this space. In 2007, Fu et al. [3] gave the central BMO estimates for commutators of $n$-dimensional fractional and Hardy operators. And recently, author $[4,5,6,7,8,9]$ also considers the boundedness for Hardy operator and its commutator in (variable exponent) Herz-Morrey spaces.

The theory of variable exponent Lebesgue spaces is started by Orlicz (see [10], 1931) and Nakano (see [11, 12], 1950 and 1951). In particular, the definition of Musielak-Orlicz spaces is clearly stated in [11]. However, the variable exponent function space, due to the failure of translation invariance and related properties, is very difficult to analyze.

Received October 8, 2012; Revised January 9, 2014.

2010 Mathematics Subject Classification. Primary 42B20; Secondary 47B38.

Key words and phrases. Herz-Morrey space, Hardy operator, Riesz potential, variable exponent, weighted estimate. 
Nowadays there is an evident increase of investigations related to both the theory of the spaces $L^{q(\cdot)}(\Omega)$ themselves and the operator theory in these spaces. This is caused by possible applications to models with non-standard local growth (in elasticity theory, fluid mechanics, differential equations, see for example [13], [14] and references therein) and is based on recent breakthrough result on boundedness of the Hardy-Littlewood maximal operator in these spaces. By virtue of the fine works [15, 16, 17, 18, 20, 21, 22, 25, 26, 27, 28], some important conditions on variable exponent, for example, the log-Hölder conditions and the Muckenhoupt type condition, have been obtained.

Now, we define the n-dimensional fractional Hardy-type operators of variable order $\beta(x)$ as follows.

Definition 1.1. Let $f$ be a locally integrable function on $\mathbb{R}^{n}, 0 \leq \beta(x)<n$. The n-dimensional fractional Hardy-type operators of variable order $\beta(x)$ are defined by

$$
\begin{aligned}
& \mathscr{H}_{\beta(\cdot)}(f)(x):=\frac{1}{|x|^{n-\beta(x)}} \int_{|t|<|x|} f(t) \mathrm{d} t, \\
& \mathscr{H}_{\beta(\cdot)}^{*}(f)(x):=\int_{|t| \geq|x|} \frac{f(t)}{|t|^{n-\beta(x)}} \mathrm{d} t,
\end{aligned}
$$

where $x \in \mathbb{R}^{n} \backslash\{0\}$.

Obviously, when $\beta(x)=0, \mathscr{H}_{\beta(\cdot)}$ is just $\mathscr{H}$, and denote by $\mathscr{H}^{*}:=\mathscr{H}_{\beta(\cdot)}^{*}=$ $\mathscr{H}_{0}^{*}$. And when $\beta(x)$ is constant, $\mathscr{H}_{\beta(\cdot)}$ and $\mathscr{H}_{\beta(\cdot)}^{*}$ will become $\mathscr{H}_{\beta}$ and $\mathscr{H}_{\beta}^{*}$ [3], respectively.

The Riesz-type potential operator of variable order $\beta(x)$ is defined by

$$
I_{\beta(\cdot)}(f)(x)=\int_{\mathbb{R}^{n}} \frac{f(y)}{|x-y|^{n-\beta(x)}} \mathrm{d} y, \quad 0<\beta(x)<n .
$$

The boundedness of the operator $I_{\beta(\cdot)}$ from the space $L^{p(\cdot)}\left(\mathbb{R}^{n}\right)$ with the variable exponent $p(x)$ into the space $L^{q(\cdot)}\left(\mathbb{R}^{n}\right)$ with the limiting Sobolev exponent

$$
\frac{1}{q(x)}=\frac{1}{p(x)}-\frac{\beta(x)}{n}
$$

was an open problem for a long time. It was solved in the case of bounded domains. First, in [29], in the case of bounded domains $\Omega$, there was proved a conditional result: the Sobolev theorem is valid for the potential operator $I_{\beta(\cdot)}$ within the framework of the spaces $L^{p(\cdot)}(\Omega)$ with the variable exponent $p(x)$ satisfying the logarithmic Dini condition, if the maximal operator is bounded in the space $L^{p(\cdot)}(\Omega)$. In 2004, Diening [20] proved the boundedness of the maximal operator.

In 2004, Diening [19] proved Sobolev's theorem for the potential $I_{\beta}$ on the whole space $\mathbb{R}^{n}$ assuming that $p(x)$ is constant at infinity $(p(x)$ is always constant outside some large ball) and satisfies the same logarithmic condition as in [29]. Another progress for unbounded domains is the result of Cruz-Uribe 
et al. [18] on the boundedness of the maximal operator in unbounded domains for exponents $p(x)$ satisfying the logarithmic smoothness condition both locally and at infinity.

In [24], Kokilashvili and Samko prove Sobolev-type theorem for the potential $I_{\beta(\cdot)}$ from the space $L^{p(\cdot)}\left(\mathbb{R}^{n}\right)$ into the weighted space $L_{\omega}^{q(\cdot)}\left(\mathbb{R}^{n}\right)$ with the power weight $\omega$ fixed to infinity, under the logarithmic condition for $p(x)$ satisfied locally and at infinity, not supposing that $p(x)$ is constant at infinity but assuming that $p(x)$ takes its minimal value at infinity.

Motivated by the above results, we are to investigate mapping properties of the fractional Hardy-type operators $\mathscr{H}_{\beta(\cdot)}$ and $\mathscr{H}_{\beta(\cdot)}^{*}$ within the framework of the Herz-Morrey spaces with variable exponent.

Throughout this paper, we will denote by $|S|$ the Lebesgue measure and by $\chi_{S}$ the characteristic function for a measurable set $S \subset \mathbb{R}^{n} ; B(x, r)$ is the ball centered at $x$ and of radius $r ; B_{0}=B(0,1)$. $C$ denotes a constant that is independent of the main parameters involved but whose value may differ from line to line. For any index $1<q(x)<\infty$, we denote by $q^{\prime}(x)$ its conjugate index, namely, $q^{\prime}(x)=\frac{q(x)}{q(x)-1}$. For $A \sim D$, we mean that there is a constant $C>0$ such that $C^{-1} D \leq A \leq C D$.

\section{Preliminaries}

In this section, we give the definition of Lebesgue and Herz-Morrey spaces with variable exponent, and give basic properties and useful lemmas.

\subsection{Function spaces with variable exponent}

Let $\Omega$ be a measurable set in $\mathbb{R}^{n}$ with $|\Omega|>0$. We first define Lebesgue spaces with variable exponent.

Definition 2.1. Let $q(\cdot): \Omega \rightarrow[1, \infty)$ be a measurable function.

(I)) The Lebesgue spaces with variable exponent $L^{q(\cdot)}(\Omega)$ is defined by $L^{q(\cdot)}(\Omega)=\left\{f\right.$ is measurable function : $F_{q}(f / \eta)<\infty$ for some constant $\left.\eta>0\right\}$, where $F_{q}(f):=\int_{\Omega}|f(x)|^{q(x)} \mathrm{d} x$. The Lebesgue space $L^{q(\cdot)}(\Omega)$ is a Banach function space with respect to the norm

$$
\|f\|_{L^{q(\cdot)}(\Omega)}=\inf \left\{\eta>0: F_{q}(f / \eta)=\int_{\Omega}\left(\frac{|f(x)|}{\eta}\right)^{q(x)} \mathrm{d} x \leq 1\right\} .
$$

(II)) The space $L_{\text {loc }}^{q(\cdot)}(\Omega)$ is defined by

$L_{\text {loc }}^{q(\cdot)}(\Omega)=\left\{f\right.$ is measurable $: f \in L^{q(\cdot)}\left(\Omega_{0}\right)$ for all compact subsets $\left.\Omega_{0} \subset \Omega\right\}$.

III) The weighted Lebesgue space $L_{\omega}^{q(\cdot)}(\Omega)$ is defined by as the set of all measurable functions for which

$$
\|f\|_{L_{\omega}^{q(\cdot)}(\Omega)}=\|\omega f\|_{L^{q(\cdot)}(\Omega)}<\infty .
$$


Next we define some classes of variable exponent functions. Given a function $f \in L_{\text {loc }}^{1}\left(\mathbb{R}^{n}\right)$, the Hardy-Littlewood maximal operator $M$ is defined by

$$
M f(x)=\sup _{r>0} r^{-n} \int_{B(x, r)}|f(y)| \mathrm{d} y,
$$

where $B(x, r)=\left\{y \in \mathbb{R}^{n}:|x-y|<r\right\}$.

Definition 2.2. Given a measurable function $q(\cdot)$ defined on $\mathbb{R}^{n}$, we write

$$
q_{-}:=\underset{x \in \mathbb{R}^{n}}{\operatorname{essinf}} q(x), \quad q_{+}:=\underset{x \in \mathbb{R}^{n}}{\operatorname{essipsup}} q(x) .
$$

(I) $q_{-}^{\prime}=\operatorname{ess}_{x \in \mathbb{R}^{n}} q^{\prime}(x)=\frac{q_{+}}{q_{+}-1}, \quad q_{+}^{\prime}=\operatorname{ess~sup}_{x \in \mathbb{R}^{n}} q^{\prime}(x)=\frac{q_{-}}{q_{--}}$.

(II) Denote by $\mathscr{P}\left(\mathbb{R}^{n}\right)$ the set of all measurable functions $q(\cdot): \mathbb{R}^{n} \rightarrow$ $(1, \infty)$ such that

$$
1<q_{-} \leq q(x) \leq q_{+}<\infty, \quad x \in \mathbb{R}^{n} .
$$

(III) The set $\mathscr{B}\left(\mathbb{R}^{n}\right)$ consists of all measurable functions $q(\cdot) \in \mathscr{P}\left(\mathbb{R}^{n}\right)$ satisfying that the Hardy-Littlewood maximal operator $M$ is bounded on $L^{q(\cdot)}\left(\mathbb{R}^{n}\right)$.

(IV) The set $\mathscr{C}_{0}^{\log }\left(\mathbb{R}^{n}\right)$ consists of all locally log-Hölder continuous functions $q(\cdot): \mathbb{R}^{n} \rightarrow(0, \infty)$ satisfies the condition

$$
|q(x)-q(y)| \leq \frac{-C}{\ln (|x-y|)}, \quad|x-y| \leq 1 / 2, x, y \in \mathbb{R}^{n} .
$$

(V) The set $\mathscr{C}_{\infty}^{\log }\left(\mathbb{R}^{n}\right)$ consists of all log-Hölder continuous at infinity functions $q(\cdot): \mathbb{R}^{n} \rightarrow(0, \infty)$ satisfies the condition

$$
|q(x)-q(\infty)| \leq \frac{C_{\infty}}{\ln (e+|x|)}, \quad x \in \mathbb{R}^{n},
$$

where $q(\infty)=\lim _{|x| \rightarrow \infty} q(x)$.

(VI) Denote by $\mathscr{C}^{\log }\left(\mathbb{R}^{n}\right):=\mathscr{C}_{0}^{\log }\left(\mathbb{R}^{n}\right) \cap \mathscr{C}_{\infty}^{\log }\left(\mathbb{R}^{n}\right)$ the set of all globally $\log$-Hölder continuous functions $q(\cdot): \mathbb{R}^{n} \rightarrow(0, \infty)$.

Remark 1. The $\mathscr{C}_{\infty}^{\log }\left(\mathbb{R}^{n}\right)$ condition is equivalent to the uniform continuity condition

$$
|q(x)-q(y)| \leq \frac{C}{\ln (e+|x|)}, \quad|y| \geq|x|, x, y \in \mathbb{R}^{n} .
$$

The $\mathscr{C}_{\infty}^{\log }\left(\mathbb{R}^{n}\right)$ condition was originally defined in this form in [18].

Next we define the Herz-Morrey spaces with variable exponent. Let $B_{k}=$ $B\left(0,2^{k}\right)=\left\{x \in \mathbb{R}^{n}:|x| \leq 2^{k}\right\}, A_{k}=B_{k} \backslash B_{k-1}$ and $\chi_{k}=\chi_{A_{k}}$ for $k \in \mathbb{Z}$.

Definition 2.3. Suppose that $\alpha \in \mathbb{R}, 0 \leq \lambda<\infty, 0<p<\infty, q(\cdot) \in \mathscr{P}\left(\mathbb{R}^{n}\right)$. The Herz-Morrey space with variable exponent $M \dot{K}_{p, q(\cdot)}^{\alpha, \lambda}\left(\mathbb{R}^{n}\right)$ is defined by

$$
M \dot{K}_{p, q(\cdot)}^{\alpha, \lambda}\left(\mathbb{R}^{n}\right)=\left\{f \in L_{\mathrm{loc}}^{q(\cdot)}\left(\mathbb{R}^{n} \backslash\{0\}\right):\|f\|_{M \dot{K}_{p, q(\cdot)}^{\alpha, \lambda}\left(\mathbb{R}^{n}\right)}<\infty\right\},
$$


where

$$
\|f\|_{M \dot{K}_{p, q(\cdot)}^{\alpha, \lambda}\left(\mathbb{R}^{n}\right)}=\sup _{k_{0} \in \mathbb{Z}} 2^{-k_{0} \lambda}\left(\sum_{k=-\infty}^{k_{0}} 2^{k \alpha p}\left\|f \chi_{k}\right\|_{L^{q(\cdot)}\left(\mathbb{R}^{n}\right)}^{p}\right)^{\frac{1}{p}} .
$$

Compare the variable Herz-Morrey space $M \dot{K}_{p, q(\cdot)}^{\alpha, \lambda}\left(\mathbb{R}^{n}\right)$ with the variable Herz space $\dot{K}_{q(\cdot)}^{\alpha, p}\left(\mathbb{R}^{n}\right)$, where

$$
\dot{K}_{q(\cdot)}^{\alpha, p}\left(\mathbb{R}^{n}\right)=\left\{f \in L_{\mathrm{loc}}^{q(\cdot)}\left(\mathbb{R}^{n} \backslash\{0\}\right): \sum_{k=-\infty}^{\infty} 2^{k \alpha p}\left\|f \chi_{k}\right\|_{L^{q(\cdot)}\left(\mathbb{R}^{n}\right)}^{p}<\infty\right\},
$$

Obviously, $M \dot{K}_{p, q(\cdot)}^{\alpha, 0}\left(\mathbb{R}^{n}\right)=\dot{K}_{q(\cdot)}^{\alpha, p}\left(\mathbb{R}^{n}\right)$.

In 2012, Almeida and Drihem [1] discuss the boundedness of a wide class of sublinear operators on Herz spaces $K_{q(\cdot)}^{\alpha(\cdot), p}\left(\mathbb{R}^{n}\right)$ and $\dot{K}_{q(\cdot)}^{\alpha(\cdot), p}\left(\mathbb{R}^{n}\right)$ with variable exponent $\alpha(\cdot)$ and $q(\cdot)$. In this paper, the author only considers Herz-Morrey space $M \dot{K}_{p, q(\cdot)}^{\alpha(\cdot), \lambda}\left(\mathbb{R}^{n}\right)$ with variable exponent $q(\cdot)$ but fixed $\alpha \in \mathbb{R}$ and $p \in(0, \infty)$. However, for the case of the exponent $\alpha(\cdot)$ is variable as well, which can be found in the furthermore work for the author.

\subsection{Auxiliary propositions and lemmas}

In this part we state some auxiliary propositions and lemmas which will be needed for proving our main theorems. And we only describe partial results we need.

Proposition 2.1. Let $q(\cdot) \in \mathscr{P}\left(\mathbb{R}^{n}\right)$.

(I) If $q(\cdot) \in \mathscr{C}^{\log }\left(\mathbb{R}^{n}\right)$, then we have $q(\cdot) \in \mathscr{B}\left(\mathbb{R}^{n}\right)$.

(II) $q(\cdot) \in \mathscr{B}\left(\mathbb{R}^{n}\right)$ if and only if $q^{\prime}(\cdot) \in \mathscr{B}\left(\mathbb{R}^{n}\right)$.

The first part in Proposition 2.1 is independently due to Cruz-Uribe et al. [18] and to Nekvinda [27], respectively. The second of Proposition 2.1 belongs to Diening [21] (see Theorem 8.1 or Theorem 1.2 in [17]).

Remark 2. Since

$$
\left|q^{\prime}(x)-q^{\prime}(y)\right| \leq \frac{|q(x)-q(y)|}{\left(q_{-}-1\right)^{2}},
$$

it follows at once that if $q(\cdot) \in \mathscr{C}^{\log }\left(\mathbb{R}^{n}\right)$, then so does $q^{\prime}(\cdot)$-i.e., if the condition hold, then $M$ is bounded on $L^{q(\cdot)}\left(\mathbb{R}^{n}\right)$ and $L^{q^{\prime}(\cdot)}\left(\mathbb{R}^{n}\right)$. Furthermore, Diening has proved general results on Musielak-Orlicz spaces.

The order $\beta(x)$ of the fractional Hardy-type operators in Definition 1.1 is not assumed to be continuous. We assume that it is a measurable function on 
$\mathbb{R}^{n}$ satisfying the following assumptions

$$
\left\{\begin{array}{c}
\beta_{0}:=\underset{x \in \mathbb{R}^{n}}{\operatorname{essinf}} \beta(x)>0, \\
\operatorname{ess} \sup _{x \in \mathbb{R}^{n}} p(x) \beta(x)<n, \\
\operatorname{ess} \sup _{x \in \mathbb{R}^{n}} p(\infty) \beta(x)<n .
\end{array}\right.
$$

In order to prove our main results, we need the Sobolev type theorem for the space $\mathbb{R}^{n}$ which was proved in ref. [24] for the exponents $p(x)$ not necessarily constant in a neigbourhood of infinity, but with some extra power weight fixed to infinity and under the assumption that $p(x)$ takes its minimal value at infinity.

Proposition 2.2. Suppose that $p(\cdot) \in \mathscr{C}^{\log }\left(\mathbb{R}^{n}\right) \cap \mathscr{P}\left(\mathbb{R}^{n}\right)$. Let

$$
1<p(\infty) \leq p(x) \leq p_{+}<\infty,
$$

and $\beta(x)$ meet condition (2.4). Then the following weighted Sobolev-type estimate is valid for the operator $I_{\beta(\cdot)}$ :

$$
\left\|(1+|x|)^{-\gamma(x)} I_{\beta(\cdot)}(f)\right\|_{L^{q(\cdot)}\left(\mathbb{R}^{n}\right)} \leq C\|f\|_{L^{p(\cdot)}\left(\mathbb{R}^{n}\right)},
$$

where

$$
\frac{1}{q(x)}=\frac{1}{p(x)}-\frac{\beta(x)}{n}
$$

is the Sobolev exponent and

$$
\gamma(x)=C_{\infty} \beta(x)\left(1-\frac{\beta(x)}{n}\right) \leq \frac{n}{4} C_{\infty},
$$

$C_{\infty}$ being the Dini-Lipschitz constant from $(2.2)$ which $q(\cdot)$ is replaced by $p(\cdot)$.

Remark 3. (i) If $\beta(x)$ satisfies the condition of type $(2.2):|\beta(x)-\beta(\infty)| \leq$ $\frac{C_{\infty}}{\ln (e+|x|)}\left(x \in \mathbb{R}^{n}\right)$, then the weight $(1+|x|)^{-\gamma(x)}$ is equivalent to the weight $(1+|x|)^{-\gamma(\infty)}$.

(ii) One can also treat operator (1.1) with $\beta(x)$ replaced by $\beta(y)$. In the case of potentials over bounded domains $\Omega$ such potentials differ unessentially, if the function $\beta(x)$ satisfies the smoothness logarithmic condition as (2.1), since

$$
C_{1}|x-y|^{n-\beta(y)} \leq|x-y|^{n-\beta(x)} \leq C_{2}|x-y|^{n-\beta(y)}
$$

in this case (see [29], p. 277).

(iii) Under the assumptions of Proposition 2.2, similar conclusion is also valid for the fractional maximal operator

$$
M_{\beta(\cdot)}(f)(x)=\sup _{r>0} \frac{1}{|B(x, r)|^{n-\beta(x)}} \int_{B(x, r)}|f(y)| \mathrm{d} y .
$$


(iv) When $p(\cdot) \in \mathscr{P}\left(\mathbb{R}^{n}\right)$, the assumption that $p(\cdot) \in \mathscr{C}^{\log }\left(\mathbb{R}^{n}\right)$ is equivalent to assuming $1 / p(\cdot) \in \mathscr{C}^{\log }\left(\mathbb{R}^{n}\right)$, since

$$
\left|\frac{p(x)-p(y)}{\left(p_{+}\right)^{2}}\right| \leq\left|\frac{1}{p(x)}-\frac{1}{p(y)}\right|=\left|\frac{p(x)-p(y)}{p(x) p(y)}\right| \leq\left|\frac{p(x)-p(y)}{\left(p_{-}\right)^{2}}\right| .
$$

And further, $1 / p(\cdot) \in \mathscr{C}^{\log }\left(\mathbb{R}^{n}\right)$ implies that $1 / q(\cdot) \in \mathscr{C}^{\log }\left(\mathbb{R}^{n}\right)$ as well.

The next lemma known as the generalized Hölder's inequality on Lebesgue spaces with variable exponent, and the proof can be found in [15].

Lemma 2.1 (Generalized Hölder's inequality). Suppose that $q(\cdot) \in \mathscr{P}\left(\mathbb{R}^{n}\right)$, then for any $f \in L^{q(\cdot)}\left(\mathbb{R}^{n}\right)$ and any $g \in L^{q^{\prime}(\cdot)}\left(\mathbb{R}^{n}\right)$, we have

$$
\int_{\mathbb{R}^{n}}|f(x) g(x)| \mathrm{d} x \leq C_{q}\|f\|_{L^{q(\cdot)}\left(\mathbb{R}^{n}\right)}\|g\|_{L^{q^{\prime}(\cdot)}\left(\mathbb{R}^{n}\right)},
$$

where $C_{q}=1+1 / q_{-}-1 / q_{+}$.

The following lemma can be found in [23].

Lemma 2.2. Let $q(\cdot) \in \mathscr{B}\left(\mathbb{R}^{n}\right)$.

(I) Then there exist positive constants $\delta \in(0,1)$ and $C>0$ such that

$$
\frac{\left\|\chi_{S}\right\|_{L^{q(\cdot)}\left(\mathbb{R}^{n}\right)}}{\left\|\chi_{B}\right\|_{L^{q(\cdot)}\left(\mathbb{R}^{n}\right)}} \leq C\left(\frac{|S|}{|B|}\right)^{\delta}
$$

for all balls $B$ in $\mathbb{R}^{n}$ and all measurable subsets $S \subset B$.

(II) Then there exists a positive constant $C>0$ such that

$$
C^{-1} \leq \frac{1}{|B|}\left\|\chi_{B}\right\|_{L^{q(\cdot)}\left(\mathbb{R}^{n}\right)}\left\|\chi_{B}\right\|_{L^{q^{\prime}(\cdot)}\left(\mathbb{R}^{n}\right)} \leq C
$$

for all balls $B$ in $\mathbb{R}^{n}$.

Remark 4. (i) If $q_{1}(\cdot), q_{2}(\cdot) \in \mathscr{C}^{\log }\left(\mathbb{R}^{n}\right) \cap \mathscr{P}\left(\mathbb{R}^{n}\right)$, then we see that $q_{1}^{\prime}(\cdot), q_{2}(\cdot) \in$ $\mathscr{B}\left(\mathbb{R}^{n}\right)$. Hence we can take positive constants $0<\delta_{1}<1 /\left(q_{1}^{\prime}\right)_{+}, 0<\delta_{2}<$ $1 /\left(q_{2}\right)+$ such that

$$
\frac{\left\|\chi_{S}\right\|_{L^{q_{1}^{\prime}(\cdot)}\left(\mathbb{R}^{n}\right)}}{\left\|\chi_{B}\right\|_{L^{q_{1}^{\prime}(\cdot)}\left(\mathbb{R}^{n}\right)}} \leq C\left(\frac{|S|}{|B|}\right)^{\delta_{1}}, \quad \frac{\left\|\chi_{S}\right\|_{L^{q_{2}(\cdot)}\left(\mathbb{R}^{n}\right)}}{\left\|\chi_{B}\right\|_{L^{q_{2}(\cdot)}\left(\mathbb{R}^{n}\right)}} \leq C\left(\frac{|S|}{|B|}\right)^{\delta_{2}}
$$

hold for all balls $B$ in $\mathbb{R}^{n}$ and all measurable subsets $S \subset B$ (see [7, 23]).

(ii) On the other hand, Kopaliani [25] has proved the conclusion: If the exponent $q(\cdot) \in \mathscr{P}\left(\mathbb{R}^{n}\right)$ equals to a constant outside some large ball, then $q(\cdot) \in \mathscr{B}\left(\mathbb{R}^{n}\right)$ if and only if $q(\cdot)$ satisfies the Muckenhoupt type condition

$$
\sup _{Q: \text { cube }} \frac{1}{|Q|}\left\|\chi_{Q}\right\|_{L^{q(\cdot)}\left(\mathbb{R}^{n}\right)}\left\|\chi_{Q}\right\|_{L^{q^{\prime}(\cdot)\left(\mathbb{R}^{n}\right)}}<\infty .
$$




\section{Main results and their proofs}

Our main result can be stated as follows.

Theorem 3.1. Suppose that $q_{1}(\cdot) \in \mathscr{C}^{\log }\left(\mathbb{R}^{n}\right) \cap \mathscr{P}\left(\mathbb{R}^{n}\right)$ satisfies condition $(2.5)$, and $\beta(x)$ meet condition $(2.4)$ which $p(\cdot)$ is replaced by $q_{1}(\cdot)$. Define the variable exponent $q_{2}(\cdot)$ by

$$
\frac{1}{q_{2}(x)}=\frac{1}{q_{1}(x)}-\frac{\beta(x)}{n}
$$

Let $0<p_{1} \leq p_{2}<\infty, \lambda \geq 0, \alpha<\lambda+n \delta_{1}$, where $\delta_{1} \in\left(0,1 /\left(q_{1}^{\prime}\right)_{+}\right)$is the constant appearing in (2.7). Then

$$
\left\|(1+|x|)^{-\gamma(x)} \mathscr{H}_{\beta(\cdot)}(f)\right\|_{M \dot{K}_{p_{2}, q_{2}(\cdot)}^{\alpha, \lambda}\left(\mathbb{R}^{n}\right)} \leq C\|f\|_{M \dot{K}_{p_{1}, q_{1}(\cdot)}^{\alpha, \lambda}\left(\mathbb{R}^{n}\right)},
$$

where $\gamma(x)$ is defined as in (2.6), and $C_{\infty}$ is the Dini-Lipschitz constant from (2.2) which $q_{1}(\cdot)$ instead of $q(\cdot)$.

Proof. For any $f \in M \dot{K}_{p, q(\cdot)}^{\alpha, \lambda}\left(\mathbb{R}^{n}\right)$, if we denote $f_{j}:=f \cdot \chi_{j}=f \cdot \chi_{A_{j}}$ for each $j \in \mathbb{Z}$, then we can write

$$
f(x)=\sum_{j=-\infty}^{\infty} f(x) \cdot \chi_{j}(x)=\sum_{j=-\infty}^{\infty} f_{j}(x) .
$$

By (1.0a) and Lemma 2.1, we have

$$
\begin{aligned}
\left|\mathscr{H}_{\beta(\cdot)}(f)(x) \cdot \chi_{k}(x)\right| & \leq \frac{1}{|x|^{n-\beta(x)}} \int_{|t|<|x|}|f(t)| \mathrm{d} t \cdot \chi_{k}(x) \\
& \leq \frac{1}{|x|^{n-\beta(x)}} \int_{B_{k}}|f(t)| \mathrm{d} t \cdot \chi_{k}(x) \\
& \leq C 2^{-k n}|x|^{\beta(x)}\left(\sum_{j=-\infty}^{k} \int_{A_{j}}|f(t)| \mathrm{d} t\right) \cdot \chi_{k}(x) \\
& \leq C 2^{-k n} \sum_{j=-\infty}^{k}\left\|f_{j}\right\|_{L^{q_{1}(\cdot)}\left(\mathbb{R}^{n}\right)}\left\|\chi_{j}\right\|_{L^{q_{1}^{\prime}(\cdot)}\left(\mathbb{R}^{n}\right)} \cdot|x|^{\beta(x)} \chi_{k}(x) .
\end{aligned}
$$

For Proposition 2.2, we note that

$$
\begin{aligned}
I_{\beta(\cdot)}\left(\chi_{B_{k}}\right)(x) & \geq I_{\beta(\cdot)}\left(\chi_{B_{k}}\right)(x) \cdot \chi_{B_{k}}(x)=\int_{B_{k}} \frac{1}{|x-y|^{n-\beta(x)}} \mathrm{d} y \cdot \chi_{B_{k}}(x) \\
& \geq C|x|^{\beta(x)} \cdot \chi_{B_{k}}(x) \geq C|x|^{\beta(x)} \cdot \chi_{k}(x) .
\end{aligned}
$$


Using Proposition 2.2, Lemma 2.2, (2.7), (3.1) and (3.2), we have

$$
\begin{aligned}
& \left\|(1+|x|)^{-\gamma(x)} \mathscr{H}_{\beta(\cdot)}(f) \cdot \chi_{k}(\cdot)\right\|_{L^{q_{2}(\cdot)}\left(\mathbb{R}^{n}\right)} \\
& \leq C 2^{-k n} \sum_{j=-\infty}^{k}\left\|f_{j}\right\|_{L^{q_{1}(\cdot)}\left(\mathbb{R}^{n}\right)}\left\|\chi_{j}\right\|_{L^{q_{1}^{\prime}(\cdot)}\left(\mathbb{R}^{n}\right)}\left\|(1+|x|)^{-\gamma(x)}|\cdot|^{\beta(x)} \cdot \chi_{k}(\cdot)\right\|_{L^{q_{2}(\cdot)}{ }_{\left(\mathbb{R}^{n}\right)}} \\
& \leq C 2^{-k n} \sum_{j=-\infty}^{k}\left\|f_{j}\right\|_{L^{q_{1}(\cdot)}\left(\mathbb{R}^{n}\right)}\left\|\chi_{j}\right\|_{L^{q_{1}^{\prime}(\cdot)}\left(\mathbb{R}^{n}\right)}\left\|(1+|x|)^{-\gamma(x)} I_{\beta(\cdot)}\left(\chi_{B_{k}}\right)\right\|_{L^{q_{2}(\cdot)}{ }_{\left(\mathbb{R}^{n}\right)}} \\
& \leq C 2 \sum_{j=-\infty}^{-k n}\left\|f_{j}\right\|_{L^{q_{1}(\cdot)}\left(\mathbb{R}^{n}\right)}\left\|\chi_{j}\right\|_{L^{q_{1}^{\prime}(\cdot)}\left(\mathbb{R}^{n}\right)}\left\|\chi_{B_{k}}\right\|_{L^{q_{1}(\cdot)}\left(\mathbb{R}^{n}\right)} \\
& \leq C 2^{-k n}\left\|\chi_{B_{k}}\right\|_{L^{q_{1}(\cdot)}} \sum_{\left(\mathbb{R}^{n}\right)}^{k}\left\|f_{j=-\infty}\right\|_{L^{q_{1}(\cdot)}\left(\mathbb{R}^{n}\right)}\left\|\chi_{B_{j}}\right\|_{L^{q_{1}^{(\cdot)}}}\left(\mathbb{R}^{n}\right) \\
& \leq C \sum_{j=-\infty}^{k}\left\|f_{j}\right\|_{L^{q_{1}(\cdot)}} \frac{\left.\| \chi_{B_{j}}\right) \|_{L^{q_{1}^{\prime}(\cdot)}\left(\mathbb{R}^{n}\right)}}{\left\|\chi_{B_{k}}\right\|_{L^{q_{1}^{\prime}(\cdot)}\left(\mathbb{R}^{n}\right)}} \\
& \leq C \sum_{j=-\infty}^{k} 2^{(j-k) n \delta_{1}}\left\|f_{j}\right\|_{L^{q_{1}(\cdot)}\left(\mathbb{R}^{n}\right)} .
\end{aligned}
$$

Because of $0<p_{1} / p_{2} \leq 1$, then we apply inequality

$$
\left(\sum_{i=-\infty}^{\infty}\left|a_{i}\right|\right)^{p_{1} / p_{2}} \leq \sum_{i=-\infty}^{\infty}\left|a_{i}\right|^{p_{1} / p_{2}}
$$

and obtain

$$
\begin{aligned}
& \left\|(1+|x|)^{-\gamma(x)} \mathscr{H}_{\beta(\cdot)}(f)\right\|_{M \dot{K}_{p_{2}, q_{2}(\cdot)}^{\alpha, \lambda}\left(\mathbb{R}^{n}\right)}^{p_{1}} \\
= & \sup _{k_{0} \in \mathbb{Z}} 2^{-k_{0} \lambda p_{1}}\left(\sum_{k=-\infty}^{k_{0}} 2^{k \alpha p_{2}}\left\|(1+|x|)^{-\gamma(x)} \mathscr{H}_{\beta(\cdot)}(f) \cdot \chi_{k}(\cdot)\right\|_{L^{q_{2}(\cdot)}\left(\mathbb{R}^{n}\right)}^{p_{2}}\right)^{p_{1} / p_{2}} \\
\leq & \sup _{k_{0} \in \mathbb{Z}} 2^{-k_{0} \lambda p_{1}}\left(\sum_{k=-\infty}^{k_{0}} 2^{k \alpha p_{1}}\left\|(1+|x|)^{-\gamma(x)} \mathscr{H}_{\beta(\cdot)}(f) \cdot \chi_{k}(\cdot)\right\|_{L^{q_{2}(\cdot)}\left(\mathbb{R}^{n}\right)}^{p_{1}}\right) .
\end{aligned}
$$

On the other hand, note the following fact

$$
\begin{aligned}
\left\|f_{j}\right\|_{L^{q_{1}(\cdot)}\left(\mathbb{R}^{n}\right)} & =2^{-j \alpha}\left(2^{j \alpha p_{1}}\left\|f_{j}\right\|_{L^{q_{1}(\cdot)}}^{p_{\left(\mathbb{R}^{n}\right)}}\right)^{1 / p_{1}} \\
& \leq 2^{-j \alpha}\left(\sum_{i=-\infty}^{j} 2^{i \alpha p_{1}}\left\|f_{i}\right\|_{L^{q_{1}(\cdot)}}^{p_{\left(\mathbb{R}^{n}\right)}}\right)^{1 / p_{1}}
\end{aligned}
$$




$$
\begin{aligned}
& =2^{j(\lambda-\alpha)}\left(2^{-j \lambda}\left(\sum_{i=-\infty}^{j} 2^{i \alpha p_{1}}\left\|f_{i}\right\|_{L^{q_{1}(\cdot)}\left(\mathbb{R}^{n}\right)}^{p_{1}}\right)^{1 / p_{1}}\right) \\
& \leq C 2^{j(\lambda-\alpha)}\|f\|_{M \dot{K}_{p_{1}, q_{1}(\cdot)}^{\alpha, \lambda}}\left(\mathbb{R}^{n}\right) .
\end{aligned}
$$

Thus, combining (3.3) and (3.5), and using $\alpha<\lambda+n \delta_{1}$, it follows that

$$
\begin{aligned}
& \left\|(1+|x|)^{-\gamma(x)} \mathscr{H}_{\beta(\cdot)}(f)\right\|_{M \dot{K}_{p_{2}, q_{2}(\cdot)}^{\alpha, \lambda}\left(\mathbb{R}^{n}\right)}^{p_{1}} \\
\leq & C \sup _{k_{0} \in \mathbb{Z}} 2^{-k_{0} \lambda p_{1}}\left(\sum_{k=-\infty}^{k_{0}} 2^{k \alpha p_{1}}\left(\sum_{j=-\infty}^{k} 2^{(j-k) n \delta_{1}}\left\|f_{j}\right\|_{L^{q_{1}(\cdot)}\left(\mathbb{R}^{n}\right)}\right)^{p_{1}}\right) \\
\leq & C\|f\|_{M \dot{K}_{p_{1}, q_{1}(\cdot)}^{\alpha, \lambda}\left(\mathbb{R}^{n}\right)}^{p_{1}} \sup _{k_{0} \in \mathbb{Z}} 2^{-k_{0} \lambda p_{1}}\left(\sum_{k=-\infty}^{k_{0}} 2^{k \alpha p_{1}}\left(\sum_{j=-\infty}^{k} 2^{(j-k) n \delta_{1}} 2^{j(\lambda-\alpha)}\right)^{p_{1}}\right) \\
\leq & C\|f\|_{M \dot{K}_{p_{1}, q_{1}(\cdot)}^{\alpha, \lambda}}^{p_{1}} \mathbb{R}^{n} \sup _{k_{0} \in \mathbb{Z}} 2^{-k_{0} \lambda p_{1}}\left(\sum_{k=-\infty}^{k_{0}} 2^{k \lambda p_{1}}\left(\sum_{j=-\infty}^{k} 2^{(j-k)\left(n \delta_{1}+\lambda-\alpha\right)}\right)^{p_{1}}\right) \\
\leq & C\|f\|_{M \dot{K}_{p_{1}, q_{1}(\cdot)}^{\alpha, \lambda}\left(\mathbb{R}^{n}\right)}^{p_{1}} \sup _{k_{0} \in \mathbb{Z}} 2^{-k_{0} \lambda p_{1}}\left(\sum_{k=-\infty}^{k_{0}} 2^{k \lambda p_{1}}\right) \\
\leq & C\|f\|_{M \dot{K}_{p_{1}, q_{1}(\cdot)}^{\alpha, \lambda}\left(\mathbb{R}^{n}\right)}^{p_{1}}
\end{aligned}
$$

Consequently, the proof of Theorem 3.1 is completed.

Theorem 3.2. Suppose that $q_{1}(\cdot) \in \mathscr{C}^{\log }\left(\mathbb{R}^{n}\right) \cap \mathscr{P}\left(\mathbb{R}^{n}\right)$ satisfies condition (2.5), and $\beta(x)$ meet condition (2.4) which $q_{1}(\cdot)$ instead of $p(\cdot)$. Define the variable exponent $q_{2}(\cdot)$ by

$$
\frac{1}{q_{2}(x)}=\frac{1}{q_{1}(x)}-\frac{\beta(x)}{n} .
$$

Let $0<p_{1} \leq p_{2}<\infty, \lambda \geq 0, \alpha>\lambda-n \delta_{2}$, where $\delta_{2} \in\left(0,1 /\left(q_{2}\right)_{+}\right)$is the constant appearing in (2.7). Then

$$
\left\|(1+|x|)^{-\gamma(x)} \mathscr{H}_{\beta(\cdot)}^{*}(f)\right\|_{M \dot{K}_{p_{2}, q_{2}(\cdot)}^{\alpha, \lambda}\left(\mathbb{R}^{n}\right)} \leq C\|f\|_{M \dot{K}_{p_{1}, q_{1}(\cdot)}^{\alpha, \lambda}\left(\mathbb{R}^{n}\right)},
$$

where $\gamma(x)$ is defined as in (2.6), and the Dini-Lipschitz constant from condition (2.2) which $q(\cdot)$ is replaced by $q_{1}(\cdot)$.

Proof. For simplicity, for any $f \in M \dot{K}_{p, q(\cdot)}^{\alpha, \lambda}\left(\mathbb{R}^{n}\right)$, we write

$$
f(x)=\sum_{j=-\infty}^{\infty} f(x) \cdot \chi_{j}(x)=\sum_{j=-\infty}^{\infty} f_{j}(x) .
$$


By (1.0b) and Lemma 2.1, we have

$$
\begin{aligned}
& \left|(1+|x|)^{-\gamma(x)} \mathscr{H}_{\beta(\cdot)}^{*}(f)(x) \cdot \chi_{k}(x)\right| \\
\leq & \int_{|t| \geq|x|} \frac{|f(t)|}{|t|^{n-\beta(x)}} \mathrm{d} t \cdot(1+|x|)^{-\gamma(x)} \chi_{k}(x) \\
\leq & C \int_{\mathbb{R}^{n} \backslash B_{k}}|f(t)||x|^{\beta(x)-n} \mathrm{~d} t \cdot(1+|x|)^{-\gamma(x)} \chi_{k}(x) \\
\leq & C \sum_{j=k+1}^{\infty} \int_{A_{j}}|f(t)||x|^{\beta(x)-n}(1+|x|)^{-\gamma(x)} \mathrm{d} t \cdot \chi_{k}(x) \\
\leq & C \sum_{j=k+1}^{\infty}\left\|f_{j}\right\|_{L^{q_{1}(\cdot)}\left(\mathbb{R}^{n}\right)}\left\|(1+|x|)^{-\gamma(x)}|\cdot|{ }^{\beta(x)-n} \chi_{j}(\cdot)\right\|_{L_{1}^{q_{1}^{\prime}(\cdot)}\left(\mathbb{R}^{n}\right)} \cdot \chi_{k}(x) .
\end{aligned}
$$

Similar to $(3.2)$, we give

$$
\begin{aligned}
I_{\beta(\cdot)}\left(\chi_{B_{j}}\right)(x) & \geq I_{\beta(\cdot)}\left(\chi_{B_{j}}\right)(x) \cdot \chi_{B_{j}}(x) \\
& =\int_{B_{j}} \frac{1}{|x-y|^{n-\beta(x)}} \mathrm{d} y \cdot \chi_{B_{j}}(x) \\
& \geq C|x|^{\beta(x)} \cdot \chi_{B_{j}}(x) \geq C|x|^{\beta(x)} \cdot \chi_{j}(x) .
\end{aligned}
$$

Since $q_{1}(\cdot) \in \mathscr{C}^{\log }\left(\mathbb{R}^{n}\right) \cap \mathscr{P}\left(\mathbb{R}^{n}\right)$ and $\beta(x)$ satisfy condition (2.4) and (2.5) which $q_{1}(\cdot)$ instead of $p(\cdot)$. So, applying Proposition 2.2, Lemma 2.2, (2.7), (3.6) and (3.7), we obtain

$$
\begin{aligned}
& \left\|(1+|x|)^{-\gamma(x)} \mathscr{H}_{\beta(\cdot)}^{*}(f) \cdot \chi_{k}(\cdot)\right\|_{L^{q_{2}(\cdot)}\left(\mathbb{R}^{n}\right)} \\
& \leq C \sum_{j=k+1}^{\infty}\left\|f_{j}\right\|_{L^{q_{1}(\cdot)}\left(\mathbb{R}^{n}\right)}\left\|\chi_{k}\right\|_{L^{q_{2}(\cdot)}\left(\mathbb{R}^{n}\right)}\left\|(1+|x|)^{-\gamma(x)}|\cdot|^{\beta(x)-n} \chi_{j}(\cdot)\right\|_{L^{q_{1}^{(\cdot)}}\left(\mathbb{R}^{n}\right)}
\end{aligned}
$$

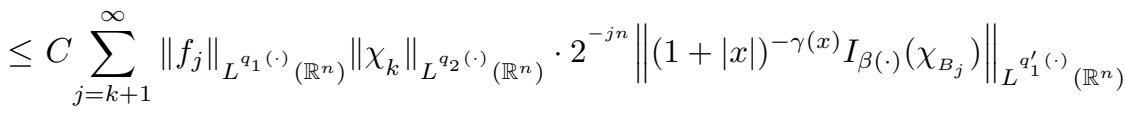

$$
\begin{aligned}
& \leq C \sum_{j=k+1}^{\infty}\left\|f_{j}\right\|_{L^{q_{1}(\cdot)}\left(\mathbb{R}^{n}\right)}\left\|\chi_{B_{k}}\right\|_{L^{q_{2}(\cdot)}\left(\mathbb{R}^{n}\right)} \cdot 2^{-j n}\left\|\chi_{B_{j}}\right\|_{L^{q_{2}^{\prime}(\cdot)}{ }_{\left(\mathbb{R}^{n}\right)}} \\
& \leq C \sum_{j=k+1}^{\infty}\left\|f_{j}\right\|_{L^{q_{1}(\cdot)}}{ }_{\left(\mathbb{R}^{n}\right)} \frac{\left\|\chi_{B_{k}}\right\|_{L^{q_{2}(\cdot)}\left(\mathbb{R}^{n}\right)}}{\left\|\chi_{B_{j}}\right\|_{L^{q_{2}(\cdot)}\left(\mathbb{R}^{n}\right)}} \\
& \leq C \sum_{j=k+1}^{\infty} 2^{(k-j) n \delta_{2}}\left\|f_{j}\right\|_{L^{q_{1}(\cdot)}\left(\mathbb{R}^{n}\right)} \text {. }
\end{aligned}
$$


Because of $0<p_{1} / p_{2} \leq 1$, therefore, applying (3.4), and combining (3.5) and (3.8), and using $\alpha>\lambda-n \delta_{2}$, it follows that

$$
\begin{aligned}
& \left\|(1+|x|)^{-\gamma(x)} \mathscr{H}_{\beta(\cdot)}^{*}(f)\right\|_{M \dot{K}_{p_{2}, q_{2}(\cdot)}^{\alpha, \lambda}\left(\mathbb{R}^{n}\right)}^{p_{1}} \\
& \leq C \sup _{k_{0} \in \mathbb{Z}} 2^{-k_{0} \lambda p_{1}}\left(\sum_{k=-\infty}^{k_{0}} 2^{k \alpha p_{1}}\left(\sum_{j=k+1}^{\infty} 2^{(k-j) n \delta_{2}}\left\|f_{j}\right\|_{L^{q_{1}(\cdot)}\left(\mathbb{R}^{n}\right)}\right)^{p_{1}}\right) \\
& \leq C\|f\|_{M \dot{K}_{p_{1}, q_{1}(\cdot)}^{\alpha, \lambda}}^{p_{1}}\left(\mathbb{R}^{n}\right) \sup _{k_{0} \in \mathbb{Z}} 2^{-k_{0} \lambda p_{1}}\left(\sum_{k=-\infty}^{k_{0}} 2^{k \alpha p_{1}}\left(\sum_{j=k+1}^{\infty} 2^{(k-j) n \delta_{2}} 2^{j(\lambda-\alpha)}\right)^{p_{1}}\right) \\
& \leq C\|f\|_{M \dot{K}_{p_{1}, q_{1}(\cdot)}^{\alpha, \lambda}}^{p_{1}} \sin ^{n} \sup _{k_{0} \in \mathbb{Z}} 2^{-k_{0} \lambda p_{1}}\left(\sum_{k=-\infty}^{k_{0}} 2^{k \lambda p_{1}}\left(\sum_{j=k+1}^{\infty} 2^{(k-j)\left(n \delta_{2}+\alpha-\lambda\right)}\right)^{p_{1}}\right)
\end{aligned}
$$

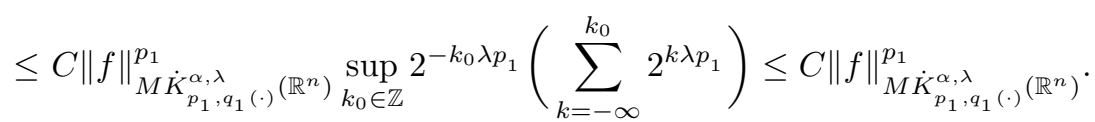

Consequently, the proof of Theorem 3.2 is completed.

In particular, when $\gamma(x)=0$ and $\beta(\cdot)$ is constant exponent, the main results above are proved by Zhang and $\mathrm{Wu}$ in [9].

Acknowledgments. The author cordially thank the referees for their valuable suggestions and useful comments which have lead to the improvement of this paper. This work was partially supported by the Fund (No.12531720) of Heilongjiang Provincial Education Department and the Project (No.SY201313) of Mudanjiang Normal University.

\section{References}

[1] A. Almeida and D. Drihem, Maximal, potential and singular type operators on Herz spaces with variable exponents, J. Math. Anal. Appl. 39 (2012), no. 2, 781-795.

[2] M. Christ and L. Grafakos, Best constants for two nonconvolution inequalities, Proc. Amer. Math. Soc. 123 (1995), no. 6, 1687-1693.

[3] Z. Fu, Z. Liu, S. Lu, and H. Wang, Characterization for commutators of $n$-dimensional fractional Hardy operators, Sci. China Ser. A 50 (2007), no. 10, 1418-1426.

[4] J. L. Wu, Boundedness of multilinear commutators of fractional Hardy operators, Acta Math. Sci. Ser. A Chin. Ed. 31 (2011), no. 4, 1055-1062.

[5] J. L. Wu and Q. G. Liu, $\lambda$-central BMO estimates for higher order commutators of Hardy operators, Commun. Math. Res. In Prss.

[6] J. L. Wu and J. M. Wang, Boundedness of multilinear commutators of fractional Hardy operators, Appl. Math. J. Chinese Univ. Ser. A 25 (2010), no. 1, 115-121.

[7] J. L. Wu and P. Zhang, Boundedness of commutators of the fractional Hardy operators on Herz-Morrey spaces with variable exponent, Adv. Math. (China), In Press.

[8] _ Boundedness of multilinear Hardy type operators on product of Herz-Morrey spaces with variable exponent, Appl. Math. J. Chinese Univ. Ser. A 28 (2013), no. 2, 154-164.

[9] P. Zhang and J. L. Wu, Boundedness of fractional Hardy type operators on Herz-Morrey spaces with variable exponent, J. Math. Practice Theory 42 (2013), no. 7, 247-254. 
[10] W. Orlicz, Über konjugierte exponentenfolgen, Studia Math. 3 (1931), no. 3, 200-212.

[11] H. Nakano, Modulared Semi-Ordered Linear Spaces, Maruzen Co. Ltd., Tokyo, 1950.

[12] _ Topology of Linear Topological Spaces, Maruzen Co. Ltd., Tokyo, 1951.

[13] M. Růžička, Electrorheological Fluids: Modeling and Mathematical Theory, New York, Springer, vol. 1748, Lecture Notes in Math., 2000.

[14] L. Diening and M. Růžička, Calderón-Zygmund operators on generalized Lebesgue spaces $L^{p(\cdot)}$ and problems related to fluid dynamics, J. Reine Angew. Math. 563 (2003), 197220.

[15] O. Kováčik and J. Rákosník, On spaces $L^{p(x)}$ and $W^{k, p(x)}$, Czechoslovak Math. J. 41 (1991), no. 4, 592-618.

[16] D. Cruz-Uribe, L. Diening, and A. Fiorenza, A new proof of the boundedness of maximal operators on variable Lebesgue spaces, Boll. Unione Mat. Ital. 2 (2009), no. 1, 151-173.

[17] D. Cruz-Uribe, A. Fiorenza, J. Martell, and C. Pérez, The boundedness of classical operators on variable $L^{p}$ spaces, Ann. Acad. Sci. Fenn. Math. 31 (2006), no. 1, 239-264.

[18] D. Cruz-Uribe, A. Fiorenza, and C. Neugebauer, The maximal function on variable $L^{p}$ spaces, Ann. Acad. Sci. Fenn. Math. 28 (2003), no. 1, 223-238.

[19] L. Diening, Riesz potential and Sobolev embeddings on generalized Lebesgue and Sobolev spaces $L^{p(\cdot)}$ and $W^{k, p(\cdot)}$, Math. Nachr. 268 (2004), 31-43.

[20] 7 (2004), no. 2, 245-253.

[21] _ Maximal functions on Musielak-Orlicz spaces and generalized Lebesgue spaces, Bull. Sci. Math. 129 (2005), no. 8, 657-700.

[22] L. Diening, P. Harjulehto, P. Hästö, Y. Mizuta, and T. Shimomura, Maximal functions in variable exponent spaces: limiting cases of the exponent, Ann. Acad. Sci. Fenn. Math. 34 (2009), no. 2, 503-522.

[23] M. Izuki, Fractional integrals on Herz-Morrey spaces with variable exponent, Hiroshima Math. J. 40 (2010), no. 3, 343-355.

[24] V. Kokilashvili and S. Samko, On Sobolev theorem for Riesz type potentials in Lebesgue spaces with variable exponent, Z. Anal. Anwendungen 22 (2003), no. 4, 899-910.

[25] T. Kopaliani, Infimal convolution and Muckenhoupt $A_{p(\cdot)}$ condition in variable $L^{p}$ spaces, Arch. Math. 89 (2007), no. 2, 185-192.

[26] A. Lerner, On some questions related to the maximal operator on variable $L^{p}$ spaces, Trans. Amer. Math. Soc. 362 (2010), no. 8, 4229-4242.

[27] A. Nekvinda, Hardy-Littlewood maximal operator on $L^{p(x)}\left(\mathbb{R}^{n}\right)$, Math. Inequal. Appl. 7 (2004), no. 2, 255-265.

[28] L. Pick and M. Růžička, An example of a space $L^{p(\cdot)}$ on which the Hardy-Littlewood maximal operator is not bounded, Expo. Math. 19 (2001), no. 4, 369-371.

[29] S. Samko, Convolution and potential type operators in $L^{p(x)}\left(\mathbb{R}^{n}\right)$, Integral Transform. Spec. Funct. 7 (1998), no. 3-4, 261-284.

Department of Mathematics

Mudanjiang Normal University

Mudanjiang, 157011, P. R. China

E-mail address: j1-wu@163.com 